\title{
A narrative review of the research status of exosomes in cardiovascular disease
}

\author{
Mingling Cui ${ }^{1 \wedge}$, Youwei Han ${ }^{2}$, Jie Yang ${ }^{3}$, Guanlan $\mathrm{Li}^{1}$, Chuanhua Yang ${ }^{3}$ \\ ${ }^{1}$ School of Traditional Chinese Medicine, Shandong University of Traditional Chinese Medicine, Jinan, China; ${ }^{2}$ Department of Cardiology, The \\ Second Affiliated Hospital of Shandong University of Traditional Chinese Medicine, Jinan, China; ${ }^{3}$ Department of Cardiology, Affiliated Hospital of \\ Shandong University of Traditional Chinese Medicine, Jinan, China \\ Contributions: (I) Conception and design: J Yang, M Cui; (II) Administrative support: C Yang; (III) Provision of study materials or patients: J Yang, C \\ Yang, Y Han; (IV) Collection and assembly of data: M Cui; (V) Data analysis and interpretation: M Cui, G Li; (VI) Manuscript writing: All authors; \\ (VII) Final approval of manuscript: All authors. \\ Correspondence to: Chuanhua Yang. The Affiliated Hospital of Shandong University of Traditional Chinese Medicine, No. 16369, Jingshi Road, Lixia \\ District, Jinan, China. Email: yangchuanhua1962@126.com.
}

Objective: Cardiovascular diseases (CVDs) are a major health problem worldwide, and medical workers are actively seeking new and effective methods to predict and treat CVDs. Exosomes are secreted by a variety of cells and exist in a variety of body fluids and tissues. Exosomes play an important role in signal transmission between cells and participate in various physiological and pathological activities of the organism. We sought to identify new diagnosis and treatment ideas related to the application of exosomes in CVDs.

Background In the cardiovascular system, exosomes are related to endothelial cells, cardiomyocytes, vascular cells, stem cells, and progenitor cells. They can promote angiogenesis, inhibit ventricular remodeling, improve heart function, inhibit local inflammation, and regulate immune responses. They play an important role in the development, injury, and disease of the cardiovascular system. However, as an emerging field, researchers are still trying to fully understand the role of exosomes and their mechanisms in mediating heart repair.

Methods: We searched the China National Knowledge Infrastructure, Wanfang Database, PubMed, and Web of Science databases to find relevant articles. The Chinese and English search terms were "exosomes" and "cardiovascular disease". Ultimately, 96 articles were included in the review.

Conclusions: Exosomes play an important role in the cardiovascular system, and are widely involved in the occurrence and development of CVDs, such as atherosclerosis (AS), acute myocardial infarction (AMI), heart failure (HF), myocardial ischemia-reperfusion (I/R) injury, pulmonary hypertension (PH), and diabetic heart disease. In this review, we summarize research on exosomes in relation to the mechanisms, diagnoses and treatments of CVDs. We also provide new diagnosis and treatment ideas, and promote the clinical application of exosomes.

Koywords! Exosomes; cardiovascular disease (CVD); mechanism; diagnosis; treatment

Submitted Oct 29, 2021. Accepted for publication Dec 30, 2021.

doi: 10.21037/apm-21-3364

View this article at: https://dx.doi.org/10.21037/apm-21-3364

\footnotetext{
$\wedge$ ORCID: 0000-0002-9181-8726.
} 


\section{Introduction}

Cardiovascular diseases (CVDs) are a major health problem worldwide. According to data from the United States (US) Centers for Disease Control and Prevention, CVDs are the leading cause of death in the US (1). According to the "China Health Statistics Yearbook 2019", CVD mortality still ranked first in 2018, and is higher than that of tumors and other diseases (2). Further, according to an epidemiological analysis, the prevalence of CVD in China continues to rise (2). As a result of societal and economic developments, especially the aging population and the acceleration of urbanization, and changes in national lifestyles, unhealthy lifestyles are becoming increasingly common, and the effects of CVD risk factors on the health of residents is becoming more and more significant. Due to the huge economic burden this place on society, the prevention and treatment of CVDs has become the focus of much medical research. Extracellular Vesicles (EV) are nano-scale membrane vesicles actively released by cells. Based on their biogenesis, size and biophysical properties, they can be further classified (for example, exosomes, microvesicles). At present, EVs, especially exosomes, have received extensive attention from researchers in the medical field because they serve as messengers for intercellular and extracellular communication. Studies have found that in the cardiovascular system, exosomes are related to endothelial cells, cardiomyocytes, vascular cells, immune cells, stem cells, and progenitor cells, which can participate in signal transmission to promote angiogenesis, inhibit ventricular remodeling, improve heart function, and inhibit local inflammation and regulate immune responses and other processes (3-5). Exosomes play an important role in the development, injury, and disease of the cardiovascular system. There increasing evidence that exosomes are related to the occurrence and development of CVDs. We systematically explained exosomes derived from cardiomyocytes, cardiac fibroblasts, endothelial cells, immune cells and other cells and their possible mechanisms of action. We also summarized the latest possible roles, diagnosis and treatment of exosomes and their contents (especially miRNA) in myocardial infarction, atherosclerosis, heart failure, myocardial ischemia reperfusion injury, pulmonary hypertension, hypertension, cardiomyopathy, and atrial fibrillation. It is hoped to bring new dawn to the diagnosis and treatment of cardiovascular diseases in the future, and to open up new ideas for the clinical application of exosomes. We present the following article in accordance with the Narrative Review reporting checklist (available at https://apm.amegroups.com/article/view/10.21037/apm-21$3364 / \mathrm{rc})$.

\section{Methods}

\section{Sources of information}

The first author retrieved relevant full-text articles (published from January 1980 to September 2021) from the China National Knowledge Infrastructure (CNKI), Wanfang Database, PubMed, and Web of Science databases. The Chinese and English search terms are "exosomes" and "cardiovascular disease". The document types retrieved included review papers and research papers.

\section{Selection criteria}

A preliminary screening of the articles' titles and abstracts was conducted. Relevant documents were then selected after extensive and intensive reading. Articles whose research content was not related to this review were excluded.

\section{Article selection/inclusion}

After the preliminary screening of the titles and abstracts of the articles, duplicate, irrelevant, and low-quality articles were removed according to the inclusion criteria. Ultimately, 96 articles were included in the review (see Figure 1).

\section{Discussion}

\section{Overview of exosomes}

Exosomes were first discovered by Pan et al. (6) in sheep reticulocytes, and were named "exosomes" by Johnstone et al. (7). Raposo (8) was the first to report that exosomes may play a role in immune responses.

Exosomes are small vesicles [diameter 30-150 nm, density $1.10-1.19 \mathrm{~g} / \mathrm{mL}(9,10)]$ surrounded by a double layer of lipid membrane, containing protein, lipid, messenger RNA (mRNA), long non-coding RNA (lncRNA), circular RNA (circRNA), microRNA (miRNA, miR), DNA, and other biological molecules $(11,12)$. Exosomes are produced by a variety of cells, such as B lymphocytes, platelets, glial cells, dendritic cells, mast cells, adipocytes, endothelial cells, smooth muscle cells, and stem cells. Certain microorganisms can also produce exosomes. Exosomes are widely distributed in blood, saliva, and urine, cerebrospinal fluid, lymphatic fluid, breast milk, amniotic 


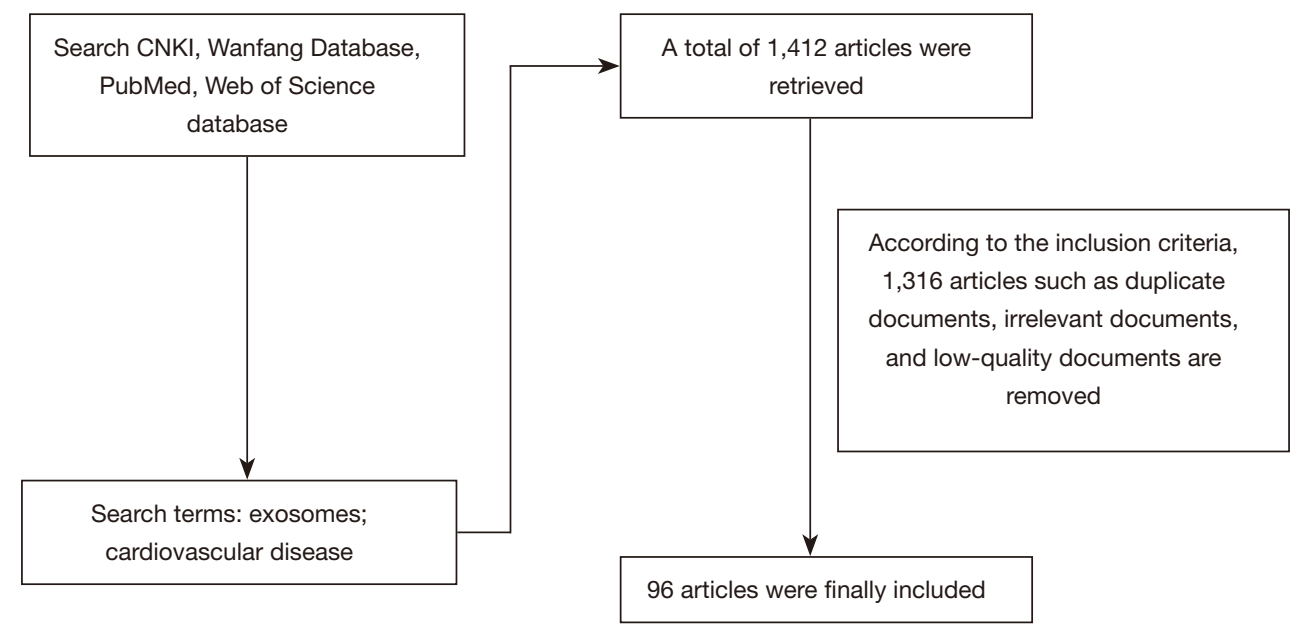

Figure 1 Source of data and information.

fluid and other body fluids $(11,13)$. Exosomes play an important role in signal transmission between cells, and are involved in various physiological and pathological activities of organisms, such as the inflammatory response, immune response, cell migration, proliferation, differentiation, apoptosis, autophagy, epithelial-mesenchymal transition, and angiogenesis. Exosomes can also be used as nanocarriers to load genes or carry drugs to target organs (11,14-16). There are more evidences show that exosomes play an important role in the cardiovascular system and are widely involved in atherosclerosis (AS), acute myocardial infarction (AMI), heart failure (HF), myocardial ischemiareperfusion (I/R) injury, pulmonary hypertension $(\mathrm{PH})$, and diabetic heart disease (17). Studies have found that in a hypoxic environment, exosomes transmit various biological information and play important biological roles by specifically binding to receptor cells. Thus, exosomes have great application potential as disease diagnostic biomarkers (18-21).

\section{Exosomes from different cell sources and cardiovascular signal transduction}

Exosomes are effective disseminators of biological signals related to myocardial function (see Table 1). Local communication in the heart is mediated by crosstalk between different cell types through EVs paracrine signaling. EVs secreted by different heart cell types may also affect the same cells that releases vesicles in an autocrine manner. Remote communication mediates functional crosstalk between the heart and other organs [e.g., the kidneys, brain, and bone marrow (BM)] by secreting EVs from the myocardium to the systemic circulation. Myocardial local and remote communication involves endocytosis, membrane fusion, or gap junction mediated transfer, and the exchange of proteins, lipids and nucleic acids through exosomes shuttles between cells. The mechanism by which exosomes work also includes receptormediated signal transduction, which regulates transcription and post-transcriptional processes in target cells. Because of these abilities, exosomes have proven to be effective disseminators of biological signals, which are involved in multiple processes that regulate cardiac function under normal cardiac physiological and pathological conditions.

\section{Exosomes secreted by cardiomyocytes}

The exosomes secreted by cardiomyocytes exert different autocrine and paracrine functions according to the content of exosomes and the type of target cells. Yu et al. (22) found that under hypoxic conditions, hypoxia inducible factor$1 \alpha(\mathrm{HIF}-1 \alpha)$ is involved in regulating the autocrine effect of tumor necrosis factor- $\alpha$ (TNF- $\alpha$ ) mediated by exosomes in cardiomyocytes. Loyer et al. (23) used a mouse model of myocardial infarction (MI), and found that AMI temporarily increases the production of cardiac EVs characterized as both exosomes and microvesicles, mainly derived from cardiomyocytes. Further, research has revealed that the EVs accumulated in the ischemic myocardium are absorbed by monocytes, which can produce interleukin-6 (IL-6) and chemokine ligands (CLs), including CCL2, CCL6, and CCL7. Notably, Ribeiro-Rodrigues et al. (24) proved that exosomes secreted by cardiomyocytes under ischemic 
Table 1 Exosomes from different cell sources and cardiovascular signal transduction

\begin{tabular}{|c|c|c|c|}
\hline Cells & Inclusions & Factor & Function \\
\hline Cardiomyocytes & miR-217 & IL-6, CCL2/6/7 & $\begin{array}{l}\text { Cardiac hypertrophy, cardiac fibrosis (enhance the proliferation of } \\
\text { fibroblasts) }\end{array}$ \\
\hline Cardiac fibroblasts & $\operatorname{miR}-21-3 p$ & Ang II & Induce cardiomyocyte hypertrophy \\
\hline \multirow{4}{*}{$\begin{array}{l}\text { Cardiac endothelial } \\
\text { cells }\end{array}$} & $\operatorname{miR}-143 / 145$ & KLF2 & Induced atherosclerotic protective phenotype \\
\hline & \multirow[t]{2}{*}{ miR-92a-3p } & $\begin{array}{l}\text { THBS1 dependent } \\
\text { mechanism }\end{array}$ & Regulating the angiogenesis \\
\hline & & $\begin{array}{l}\text { ERK1/2 MAPK signaling } \\
\text { pathway }\end{array}$ & Resistance to simulated I/R injury \\
\hline & VCAM1 & - & Regulate the local inflammatory response \\
\hline Smooth muscle cells & - & $\begin{array}{l}\text { coagulation protein } \\
\text { prothrombin }\end{array}$ & Regulate blood coagulation and calcification \\
\hline $\begin{array}{l}\text { Immune cells (dendritic } \\
\text { cells) }\end{array}$ & - & CD4(+) T cells & Promote wound healing after MI \\
\hline $\begin{array}{l}\text { Immune cells } \\
\text { (macrophages) }\end{array}$ & mir-155 & - & Inhibited the proliferation of fibroblasts and enhanced inflammation. \\
\hline Other heart-derived & $\operatorname{miR}-21-5 p$ & PTEN/Akt pathway & Promotes angiogenesis and cardiomyocyte survival \\
\hline
\end{tabular}

HIF-1 $\alpha$, hypoxia inducible factor- $1 \alpha$; TNF- $\alpha$, tumor necrosis factor- $\alpha$; IL-6, interleukin-6; CCL2/6/7, CC-motif chemokine ligands 2/6/7; Ang II, Angiotensin II; 16K PRL, 16-kDa N-terminal prolactin fragment; KLF2, Kruppel-like factor 2; THBS1, thrombospondin 1; ERK1/2, extracellular signal-regulated kinase1/2; MAPK, mitogen-activated protein kinase; I/R, ischemia-reperfusion; VCAM1, vascular cell adhesin 1; CD4, cluster of differentiation 4; MI, myocardial infarction; PTEN, gene of phosphate and tension homology deleted on chromosome ten; Akt, Protein kinase B, also known as PKB or Rac; AT1Rs, Ang II receptor type 1; iNOS, inducible nitric oxide synthase; NO, nitric oxide; CXCR4, C-X-C motif chemokine receptor type 4.

conditions promote cardiac angiogenesis. Further, research has found that miR-222 and miR-143 are the most relatively abundant miRs in ischemic exosomes, which partially explains the angiogenesis process of exosomes. Additionally, Nie et al. (25) showed that miR-217 is involved in the process of cardiac hypertrophy and cardiac fibrosis by regulating phosphatase and tensin homolog (PTEN), Thus, miR-217 is a promising therapeutic target for congestive HF. Importantly, exosomes containing miR-217 secreted by cardiomyocytes enhance the proliferation of fibroblasts in vitro (25).

\section{Exosomes derived from cardiac fibroblasts}

Cardiac fibroblast-derived exosomes have been shown to regulate various aspects of cardiomyocyte biology, such as hypertrophy. Bang et al. (26) found that cardiac fibroblasts secrete miRNA-rich exosomes, and identified miR-21$3 p$ derived from fibroblasts as an effective paracrine RNA molecule that can induce cardiomyocyte hypertrophy. Lyu et al. (27) showed that angiotensin II (Ang II) stimulates cardiac fibroblasts to release exosomes, which in turn induces the production of Ang II and its receptor expression 
in cardiomyocytes, thereby promoting pathological cardiomyocyte hypertrophy.

\section{Exosomes derived from cardiac endothelial cells}

Extensive research has shown that cardiac endothelial cells are an important source of exosomes in healthy and diseased hearts $(23,28-35)$. van Balkom et al. (28) found that endothelial cell-derived exosomes containing miR-214 inhibit the senescence of human and mouse endothelial cells and induce angiogenesis. Halkein et al. (29) found that the $16-\mathrm{kDa}$-terminal prolactin fragment (16K PRL) associated with perinatal cardiomyopathy stimulates endothelial cells to secrete exosomal miR-146a, and endothelial cell-derived exosomes are used by cardiomyocytes. The exosomal miR146a mediates the post-transcriptional gene silencing of target genes Erbb4, Notch1, and Irak1, reduces the metabolic activity of cardiomyocytes. Hergenreider et al. (30) found that EVs secreted by human umbilical vein endothelial cells (HUVECs) transduced by Kruppel-like factor 2 (KLF2) or under shear stress were rich in miR-143/145, and induced atherosclerotic protective phenotype in cocultured smooth muscle cells. AS protects the phenotype. In addition, Liu et al. (31) showed that atherosclerotic conditions promote the packaging of functional miR$92 \mathrm{a}-3 \mathrm{p}$ into endothelial microvesicles, thereby regulating the angiogenesis of receptor endothelial cells through a thrombospondin 1-dependent mechanism. Davidson et al. (32) proved that exosomes derived from endothelial cells endow cardiomyocytes with resistance to simulated I/R injury by activating the ERK1/2 MAPK signaling pathway. Hu et al. (33) demonstrated the pleiotropic effects of Mst1rich exosomes released by endothelial cells on inhibiting autophagy, promoting cell apoptosis, and inhibiting glucose metabolism in cardiomyocytes.

In CVDs, exosomes derived from endothelial cells and immune cells are becoming key players in different stages of disease development (34). The endothelial cells and cardiomyocyte-derived exosomes produced locally after MI are absorbed by the infiltrated monocytes and regulate the local inflammatory response (23). In this case, endothelialderived $\mathrm{EVs}$ are rich in the vascular cell adhesin 1, which is localized in splenic monocytes in MI (35).

\section{Other cardiac exosomes}

Qiao et al. (4) compared the therapeutic effects of exosomes from healthy donor hearts and HF hearts, and found that the pathological condition of HF changed the miRNA composition of heart-derived exosomes and impaired their regeneration activities. Further, research has shown that miR-21-5p promotes angiogenesis and cardiomyocyte survival through the PTEN/Akt pathway, thereby promoting exosomal-mediated cardiac repair (4).

Interestingly, recent studies have determined that exosomes are mediators of various forms of cardiovascular calcification in hyperphosphatemia and inflammatory environments. The exosomes released by smooth muscle cells, valvular mesenchymal cells, endothelial cells, and macrophages may promote or inhibit calcification, depending on the phenotype of their parental cells and the extracellular environment in which they are secreted (36). Kapustin et al. (37) reported that the coagulation protein prothrombin binds to the surface of exosomes derived from vascular smooth muscle cells (VSMCs), and also loads into exosomes through cell internalization to regulate blood coagulation and calcification.

Another potentially important source of exosomes in the heart is immune cells, such as dendritic cells or macrophages, which mediate immune responses to vesicle secretion. Liu et al. (38) found that the exosomes of dendritic cells from infarcted myocardium can mediate the activation of cluster of differentiation $4(\mathrm{CD} 4)^{+} \mathrm{T}$ cells through endocrine mechanisms, promote wound healing after MI, and improve cardiac function after MI. Wang et al. (39) found that miR-155-containing exosomes secreted by activated macrophages inhibited the proliferation of target cardiac fibroblasts and enhanced inflammation during cardiac injury.

\section{Circulating exosomes}

The communication mediated by cardiac exosomes has also been described in the remote microenvironment. In this respect, Pironti et al. (40) conducted research on mice subjected to pressure overload, and found that cardiomyocytes secrete Ang II receptor type 1 (AT1Rs) into circulation through exosomes. Further, studies have shown that functional AT1Rs are carried by exosomes elsewhere in circulation, providing a new paradigm for the transportation of traditional $G$ protein-coupled receptors at the whole organism level. In addition, as a result of the exogenous administration of AT1R-rich exosomes to target cardiomyocytes, skeletal muscle cells, and mesenteric resistance vessels, AT1R knockout mice displayed a blood pressure response to Ang II infusion, which provides functional evidence that exosomes significantly improve blood pressure responsiveness. Li et al. (18) studied the effects of coronary artery serum exosomes, and compared 
the exosomes of healthy controls to those of patients with myocardial ischemia (isc-Exo), and found that the miR939-5p levels of isc-Exo were significantly downregulated. Further, studies have shown that exosomes from patients with myocardial ischemia promote angiogenesis through the miR-939-iNOS-NO pathway (18). Importantly, research has shown that cardiomyocytes, but not cardiac fibroblasts or endothelial cells, may be the source of bioactive exosomes in coronary artery serum (18). Hou et al. (41) discovered a new endogenous cardioprotective mechanism; that is, that the circulating exosomes derived from longterm exercise protect the heart from myocardial I/R injury through exosomal miR-342-5p.

Interest in understanding the signal exchange between myocardium and $\mathrm{BM}$ continues to increase, and it is thought that exosomes may play an important role in these regulatory mechanisms. Cheng et al. (42) showed that after AMI, circulating myocardial miRNA (myo-miR) is carried in exosomes and mediates functional crosstalk between the ischemic heart and BM. Further, research has shown that exosomes mediate the transfer of myo-miRs to BM monocytes, such that myo-miRs downregulate CXCR4 expression and regulate the number of circulating progenitor cells. Thus, the myo-miRs carried in circulating exosomes may allow systemic responses to cardiac injury and could be used for cardiac repair.

We summarized the possible mechanisms of exosomes in cardiovascular pathophysiology to further support the potential applications of exosomes as biomarkers and therapeutics for CVDs.

\section{Exosomes and CVD}

\section{AS}

AS is a chronic inflammatory disease. Many studies have shown that exosomes play a role in various stages in the occurrence and development of AS, and play a key role in mediating inflammation, oxidative stress, and apoptosis in AS (43-51). Studies have shown that exosomes from different sources can participate in the occurrence and development of AS through the NF- $\mathrm{KB}$ signaling pathway (43-45). Yao et al. (43) found that the overexpression of peripheral platelet exosomal (PLT-exosomes) miR-25-3p inhibited coronary vascular endothelial cell inflammation. Further studies found that the inhibitory effect of PLT-exosomes carrying miR-25$3 p$ is related to the NF- $\kappa B$ signaling pathway (43). Zhong et al. (44) confirmed that exosomes derived from mature dendritic cells (mDC-exosomes) are involved in endothelial inflammation and AS. The mechanism is that $\mathrm{mDC}$ exosomes transfer miR-146 to HUVECs. Activating the NF$\kappa \mathrm{B}$ signaling pathway increases the endothelial expression of adhesion molecules, and protects HUVECs from secondary stimulation by inhibiting IL-1 receptor related kinases, which indicates that $\mathrm{mDC}$-exosomes are involved in a negative feedback loop for inflammation regulation. Bouchareychas et al. (45) found that in exosomes (BMDM-exosomes) produced by IL-4 polarized naive BM-derived macrophages, the expression of the anti-inflammatory factor miRNA$99 \mathrm{a} / 146 \mathrm{~b} / 378$ is increased. Exosomal miRNA inhibits inflammation and promotes $\mathrm{M} 2$ polarization in receptor macrophages by targeting NF- $\mathrm{BB}$ and TNF- $\alpha$ signaling. Therefore, BMDM-IL-4-exosomes may represent an effective treatment for AS and other inflammatory diseases. As the above-mentioned studies show, exosomes participate in the regulation of inflammation through the NF- $\kappa B$ pathway and affect the progression of AS.

Oxidized low density lipoprotein (ox-LDL) plays an important role in the occurrence and development of AS by stimulating macrophages and endothelial cells. Zhang et al. (46) found that exosomal miR-146a (derived from oxLDL-treated macrophages) promotes the formation of neutrophil extracellular traps (NETs) by inducing oxidative stress, intravenous administration of oxLDL-treated THP-1 cells-derived exosomes into AS mice significantly deteriorated AS. Chen et al. (47) found that exosomal miR505 from vascular endothelial cells (VECs) treated with oxLDL aggravated AS by inducing the formation of NETs. Li et al. (48) found that the reduction of exosomal MALAT1 in vascular endothelial cells treated with ox-LDL can induce the maturation of dendritic cells and promote the development of AS.

Another study by Li et al. (49) found that mesenchymal stem cell (MSC) exosomes improved AS in ApoE mice and promoted $\mathrm{M} 2$ macrophages polarization in plaques through the miR-let7/HMGA2/NF-кB pathway. In addition, MSC-derived exosomes inhibited macrophage infiltration through the miR-let7/IGF2BP1/PTEN pathway in plaques. This discovery expands our understanding of the influence of MSC-derived exosomes on atherosclerotic plaque inflammation and provides a potential method for preventing AS. Lin et al. (50) found that the transfer of miR-203-3p from dendritic cell-derived exosomes (DEXs) to BM-derived macrophages resulted in the inhibition of cathepsin S expression and AS-related phenotypes in BMderived macrophages. In attempts to reduce the progress of AS in mice, research has shown that this process involves 
the p38/MAPK signaling pathway, which has become a promising clinical target for AS. Liu et al. (51) extracted exosomes from perivascular adipose tissue (PVAT) and subcutaneous adipose tissue of wild-type C57BL/6J mice. After co-incubation, exosomes were absorbed by RAW264.7 cells. The oil red O staining and flow cytometry showed that PVAT-exosomes improve the formation of macrophage foam cells and intracellular lipid accumulation, and significantly reduce the uptake of macrophages on fluorescently labeled ox-LDL and promotes the outflow of cholesterol induced by high-density lipoprotein. In addition, a Western blot analysis showed that the macrophage scavenger receptor A was downregulated and the ATP-binding cassette transporter A1 and ATPbinding cassette transporter G1 were upregulated, which may be caused by the excessive activation of the peroxisome proliferator-activated receptor $\gamma$. Expression is mediated and does not depend on the liver $\mathrm{X}$ receptor alpha. The above studies indicate that PVAT-exosomes reduce the formation of macrophage foam cells by regulating the expression of cholesterol transporters, which provides a new mechanism by which PVAT can protect the vasculature from AS (51).

\section{AMI}

MI is myocardial necrosis caused by acute and persistent ischemia and hypoxia in the coronary arteries, and is the main cause of death in cardiovascular patients. Studies have shown that exosomes play an important role in the diagnosis and treatment of AMI. Stem cell exosomal miRs (52), including miR-19a, mirR-21, miR-21-5p, miR-21-a 5 , miR-22, miR-24, miR-26a, miR-29, miR-125b-5p, miR126, miR-201, miR-210, and miR-294, have been shown to have cardioprotective effects by enhancing the survival and function of cardiomyocytes and reducing cardiac fibrosis. In addition, the exosomal miRs of MSCs, including miR126, miR-210, miR-21, miR-23a-3p, and miR-130a-3p, induce ischemic heart damage after MI. Angiogenesis exerts a cardioprotective effect. Peng et al. (53) found that miR25-3p exosomes from MSCs can reduce myocardial cell apoptosis and inflammation by targeting pro-apoptotic genes and $E Z H 2$ to produce a cardioprotective effect and reduce MI. Zhu et al. (54) showed that exosomes derived from adipose-derived stem cells (ADSCs) promote angiogenesis in the ischemic hind limbs and heart of mice through the miRNA-31/FIH1/HIF-1 $\alpha$ pathway to improve MI. Pan et al. (55) isolated exosomes from wild or miR-146a-modified ADSCs, and evaluated the therapeutic effects of exosomes in a rat $\mathrm{AMI}$ model using hypoxia-induced $\mathrm{H} 9 \mathrm{c} 2$ model cells, and found that exosomes containing miR-146a had a greater effect than the wild exosomes treatment group in inhibiting AMI-induced apoptosis, inflammation, and fibrosis. The study also found that exosomal miR-146a reduces myocardial injury induced by AMI by downregulating the

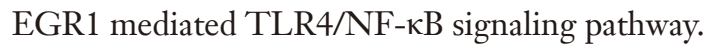

Notably, miRNA can be isolated from the blood and provide a potential source of novel diagnostic and prognostic biomarkers for CVDs. Shi et al. (56) found that human umbilical cord MSC-exosomes promote the differentiation of fibroblasts into myofibroblasts in an inflammatory environment after MI, inhibit ventricular remodeling after infarction, and protect cardiomyocytes. Santoso et al. (57) found that the exosomes secreted by induced pluripotent stem cells and their differentiated cardiomyocytes improve post-MI by regulating the autophagy of hypoxic cardiomyocytes, reducing apoptosis and fibrosis. Heart function to achieve cell-free, patient-specific treatment of ischemic cardiomyopathy. Youn et al. (58) used miR programming to modify cardiac progenitor cell (CPC)-derived exosomes to enhance angiogenesis, which may be an effective treatment strategy for the treatment of ischemic CVD. Under mild ischemic stress conditions, autophagy can produce ATP by degrading damaged organelles, thereby maintaining cell viability; however, under severe ischemic conditions, it can promote cell death and worsen heart function. When tested in animal models of MI, MSCs have cardioprotective effects.

Yu et al. (59) used Western blot analysis and quantitative real-time polymerase chain reaction (qRT-PCR) to prove that heat shock protein 20 mediated cardiomyocyte-derived exosomes activated the protein kinase B (Akt) signally pathway, inhibited TNF- $\alpha$ and IL- $1 \beta$ factors, inhibited cardiomyocyte apoptosis, and reduced MI. Chen et al. (60) conducted a qRT-PCR and found that exosomes derived from remote ischemic regulation (RIC) significantly increased the expression of angiogenesis-related molecules, such as eNOS, iNOS, HIF-1 $\alpha$, Ang-1, and VEGF. RIC exosomes improved cardiac function after $\mathrm{MI}$ and protects the heart by participating in cell apoptosis, inflammation, angiogenesis, and cardiac remodeling. Zhang et al. $(61,62)$ developed a new drug delivery system of DEXs combined with alginate hydrogels (DEXs-Gel), and found that DEXsGel continuously releases DEXs and prolongs the retention time of DEXs. They then applied DEXs-Gel to a MI mice model, and found that DEXs-Gel significantly enhanced the therapeutic effect of DEXs in improving cardiac function after MI. Flow cytometry and immunofluorescence 
staining showed that in-vitro and in-vivo DEXs significantly upregulated the infiltration of regulatory $\mathrm{T}$ (Treg) cells and M2 macrophages into the border zoom after MI, and DEXs activated Treg cells and shifted macrophages to reparative M2 macrophages. Further, studies have found that exosomes derived from dendritic cells improve heart function by activating Treg cells and affecting the polarization of macrophages after MI $(61,62)$.

\section{HF}

$\mathrm{HF}$ is the terminal state of a variety of CVDs, with pathological manifestations, such as cardiomyocyte hypertrophy and myocardial fibrosis. Many studies have shown that exosomes affect the development of HF by participating in processes such as apoptosis, myocardial fibrosis, and angiogenesis (63). Kurtzwald-Josefson et al. (63) experimentally confirmed that some miRs in cardiac fibroblast-induced pluripotent stem cells change, especially miR22, which is a key regulator of cardiac hypertrophy and remodeling. Studies have shown that CFiPSCs and their exosomes are a potential source of cardiac recovery induction, and the reduction of miR22 levels indicates that CF-iPSC exosomes have no congestive heart cell memory, making them a potential biological source for the future treatment of damaged myocardium.

Chen et al. (64) found that exosomes derived from BM-MSCs significantly protect the myocardium from cardiac hypertrophy, attenuate myocardial cell apoptosis and fibrosis, and protect cardiac function during pressure overload. Further, research has shown that MSC-derived exosomes also prevent Ang II-stimulated cell hypertrophy. Conversely, exosomes promote the premature senescence of myofibroblasts in vitro, indicating that they have antifibrosis effects in cardiac remodeling. Exosomes protect cardiomyocytes from pathological hypertrophy and may provide a promising future treatment for HF. Qiao et al. (4) found that miR-21-5p, which is derived from the exosomes of $\mathrm{HF}$, promotes angiogenesis and cardiomyocyte survival through the PTEN/Akt pathway, thereby promoting exosomes-mediated heart repair.

\section{Myocardial I/R injury}

Exosomes from different sources can reduce myocardial I/R injury by regulating apoptosis, autophagy, and inflammation. Chen et al. (65) found that exosomes derived from BMMSCs carrying miR-125b increase cell viability and reduce the apoptosis rate by targeting SIRT7, thereby reducing the area of MI and inhibiting myocardial I/R injury.
Chen et al. (66) showed that MSCs and the derived exosomal miR-143-3p inhibit myocardial I/R injuryinduced apoptosis and autophagy through the CHK2Beclin2 pathway. Zhao et al. (67) found that MSC-derived exosomes used miR-182 to change the polarization state of macrophages and inhibit myocardial inflammation to reduce myocardial I/R injury in mice. Youn, Chen, Wang and others $(58,68,69)$ found that in acute I/R injury models, a direct injection of exosomes derived from CPCs into the mouse heart can prevent cell apoptosis. Zhang et al. (70) found that serum exosomes (IPC-exosomes) may improve hemodynamics and the production of inflammatory factors and reduce apoptosis by activating the PI3K/AKT signaling pathway to reduce I/R injury. Dai et al. (71) showed that exosomes derived from M2 macrophage exosomes carry miR-148a, which reduces myocardial I/R injury by downregulating TXNIP and inactivating the TLR4/NF$\kappa \mathrm{B} / \mathrm{NLRP} 3$ inflammasome signaling pathway.

In addition, Tang et al. (72) found that MSC-exosomes protect myocardium from I/R injury by inhibiting pyrolysis. Luo et al. (73) showed that cardiac fibroblasts participate in cardioprotection through exosomal/ microbubble pathways in the acute phase of I/R injury, and post-ischemia treatment can upregulate cardiac fibroblasts exosomal/microbubble miRs-423-3p expression, and that targeting the downstream effector RAP2C can enhance this effect.

\section{PH}

PH refers to an abnormal pathophysiological state in which pulmonary artery pressure is abnormally increased and there are hemodynamic changes. Exosomes affect the progression of $\mathrm{PH}$ by participating in pulmonary artery endothelial cell (PAEC) apoptosis and pulmonary artery smooth muscle cell (PASMC) proliferation. Zhang et al. $(74,75)$ conducted in-vitro experiments, and found that MSC-exosomes significantly reduce the right ventricular systolic pressure and the right ventricular hypertrophy index in MCT-PH rats, and reduce pulmonary vascular remodeling and pulmonary fibrosis. Further, MSC-exosome significantly inhibits hypoxia-induced PAEC apoptosis and PASMC proliferation. Further studies have shown PH may be prevented from vascular remodeling via the regulation of the Wnt5a/BMP signaling pathway (74). This provides strong evidence for the treatment of $\mathrm{PH}$ by MSC-exosomes, and more importantly, these results provide a theoretical basis for the clinical prevention and treatment of $\mathrm{PH}$. Zhang et al. (76) found that hypoxia-induced 15-LO2/15-HETE 
plays an important role in $\mathrm{PH}$, and $15-\mathrm{LO} 2$ is abundantly expressed and enriched in exosomes secreted by hypoxic PAECs. In-vivo and in-vitro experiments have shown that exosomal 15-LO2 mediates hypoxia-induced PH.

Sindi et al. (77) showed that decreased KLF2 signaling is a common feature of human $\mathrm{PH}$. KLF2 regulates exosomal miRNA, miR-181a-5p, and miR-324-5p to attenuate pulmonary vascular remodeling. The mechanism is mediated by Notch4, ETS1, and other key regulators of vascular homeostasis. miR-181a-5p and miR-324-5p reduce the proliferation and angiogenesis of patient-derived cells, and slow down the disease progression of $\mathrm{PH}$ mice. This study highlights the potential therapeutic role of KLF2regulated exosomal miRNAs in $\mathrm{PH}$ and other diseases related to vascular remodeling.

\section{Hypertension}

Hypertension is a major health problem worldwide, and it is also a common risk factor for CVD morbidity and mortality. The migration of VSMCs is key to the remodeling of hypertensive blood vessels, and adventitia fibroblasts (AFs) play an important role in the homeostasis of vascular structures. Tong et al. (78) obtained primary VSMCs and AFs from the aorta of spontaneously hypertensive rats (SHRs) and Wistar-Kyoto (WKY) rats, and found that AF exosomes from SHRs convert Angconverting enzyme transfer to VSMCs, thereby increasing the level of Ang II and activating the Ang II type 1 receptor (ATR) in VSMCs, thereby promoting VSMC migration. Otani et al. (79) proved that plasma exosomes can regulate the systemic blood pressure and the structure and function of cardiovascular tissue in normotensive and hypertensive rats. Zhu et al. (80) found that the protein platelet and endothelial cell adhesion molecule 1 (PECAM1) is an EV in the blood of hypertensive patients (HBPP). Compared to the healthy control group, the morphology of EVs in the hypertension group was more obvious, as was the protein expression of PECAM1. The vesicles of HBPP are rich in exosomes, but there are no EVs in the blood samples of healthy controls. In addition, the linear regression showed that the expression of PECAM1 and the systolic blood pressure of hypertensive patients had a linear correlation $(\mathrm{R}=0.901 ; \mathrm{P}<0.01)$. Thus, the exosomes of PECAM1 protein are potential risk markers in HBPP.

Perez-Hernandez et al. (81) collected exosomes from a group of urine specimens of hypertensive patients with $(n=24)$ or without albuminuria $(n=28)$, and used 20 healthy volunteers as a control group. Their results indicate that
miRNAs are enriched in the urine exosomes of hypertensive patients, and the low expression of miR-146a in exosomes is related to the presence of albuminuria. Thus, urine exosomal miR-146a is a potential marker of proteinuria in essential hypertension. Perez-Hernandez et al. (82) also examined the changes in miRNA levels of 4 exosomes by qRT-PCR, and found that they were related to hypertensive albuminuria. Notably, exosomal miR-26a appears to play a key role in the regulation of TGF- $\beta$, and was found to be downregulated in both types of exosomes compared to healthy controls and hypertensive normoalbuminurics $(\mathrm{P}<0.01)$. Similarly, miR-26a levels were found to decrease in podocyte-derived exosomes after TGF- $\beta$ stress, and TGF- $\beta$ is a related effector of podocyte injury. These findings support the use of exosomal miRNA as a biomarker of cardiovascular risk progression and a treatment tool for early kidney injury.

\section{Atrial fibrillation (AF)}

$\mathrm{AF}$ is a common arrhythmia, which can cause serious complications such as HF and stroke. At present, there are relatively few studies on exosomes and $\mathrm{AF}$, and the studies that have been conducted have mainly focused on the diagnosis of AF. Atrial fibrosis plays a key role in AF. A key event in the pathogenesis of fibrosis is the activation of myofibroblasts by fibroblasts. Wei et al. (83) confirmed that there is a difference in the expression of miRNA derived from the plasma of patients with $\mathrm{AF}$ and normal sinus rhythm. A target gene enrichment analysis showed that the 20miRNA target genes that were significantly upregulated were mainly enriched in biological processes, such as gene expression, inflammation, energy metabolism, lipid metabolism, and enzyme activity. Mun et al. (84) found that serum exosomal miRNA and its target genes are involved in atrial function and structure, oxidative stress, and fibrosis pathways. This suggests that exosomal miRNAs may be used as novel biomarkers to reflect the progression of AF. Li et al. (85) found that exosome-mediated crosstalk between myofibroblasts and cardiomyocytes increased the susceptibility to AF by reducing the expression of Cav1.2 in cardiomyocytes.

Liu et al. (86) showed that exosomal miR-320d is related to AF cardiomyocyte apoptosis and cell viability. Further, studies have shown that STAT3 is downregulated in $\mathrm{AF}$ cardiomyocytes and is a direct target gene of miR320d. This research provides a new understanding of the molecular basis of $\mathrm{AF}$ and provides insights into treatment strategies for $\mathrm{AF}$. 


\section{Diabetic cardiomyopathy (DCM)}

Studies have shown that adults with diabetes have higher all-cause mortality than adults without diabetes (87). The increase in mortality caused by CVD is the most obvious. Some diabetic patients may develop DCM in the later stage. DCM may progress to HF, arrhythmia, and cardiogenic shock. DCM has the characteristics of myocardial microvascular endothelial cell (CMEC) injury, cardiomyocyte dysfunction, and myocardial steatosis. The cell communication between CMEC and cardiomyocytes mediated by exosomes has an emerging role in the pathogenesis of DCM, but its underlying mechanism remain unclear. Hu et al. (33) showed that under diabetic conditions, exosomes derived from CMECs are absorbed by cardiomyocytes and increase the content of the macrophage stimulating 1 (Mst1) protein, which inhibits autophagy and promotes cardiomyocyte apoptosis, reduces the interaction between death domain associated protein (Daxx) and glucose transporter type 4 (GLUT4) and enhances the association between Mst1 and Daxx to destroy GLUT4 membrane translocation, thereby inhibiting glucose uptake in diabetic conditions. Govindappa et al. (88) found that the diabetic environment activates the expression of $\mathrm{HuR}$ in the heart and cultured BM-derived macrophages (BMMØ), and stimulates the transfer of HuR to exosomes. Exosomes from macrophages exposed to a diabetic environment (high glucose or db/ $\mathrm{db}$ mice) significantly increased the inflammatory and profibrotic responses of mouse fibroblasts in vitro and cardiac fibrosis, and HuR may reduce macrophage dysfunction and pathological fibrosis in diabetes. In diabetic mice, compared with the administration of control exosomes, reconstitution with ВММØ-derived $\mathrm{HuR}$-deficient exosomes after macrophage depletion inhibits the Ang II-induced cardiac fibrosis response and preserves left ventricular function. Lin et al. (89) found that exosomes derived from MSCs can improve myocardial damage and fibrosis induced by diabetes mellitus (DM) by inhibiting the TGF- $\beta 1 /$ Smad 2 signaling pathway.

\section{Viral myocarditis}

Myocarditis is an inflammatory lesion of the myocardium, and the most common cause of myocarditis is viral infection. Studies have found that exosomes play a role in the treatment of viral myocarditis by the process of inhibiting cell apoptosis and reducing the production of pro-inflammatory factors. Gu et al. (90) found that human umbilical cord MSC-derived exosomes (hucMSC-exosomes) reduces cardiomyocyte apoptosis by activating the AMPK/ mTOR-mediated autophagy flux pathway, thereby reducing Coxsackie virus B3 (CVB3) induced myocarditis, reduces myocardial damage, reduces the production of proinflammatory cytokines, and improves heart function. These findings will be beneficial to future MSC-exosome treatments of myocarditis. Li et al. (91) examined the antiapoptotic effect of exosomes secreted by CPCs in CVB3infected cells and rats through in-vitro experimental studies. Further studies showed that CPC-exosomes eliminates the proliferation of CVB3 and regulates mTOR (91). The signaling pathway and the expression of $B c l-2$ and the caspase family have an anti-apoptotic effect in CVB3 infected cells. Thus, CPCs-exosomes may be effective tools for the treatment of viral myocarditis.

\section{Other CVDs}

The experimental results of Chen et al. (92) showed that serum exosomal miR-155-5p reduces rheumatic heart disease (RHD)-induced valve damage by inhibiting the S1PR1, SOCS1/STAT3 and IL-6/STAT3 signaling pathways. Luo et al. (93) identified $231 \mathrm{lncRNAs}$ and 179 mRNAs differentially expressed in circulating exosomes collected from patients with RHD. Through Gene Ontology and Kyoto Encyclopedia of Genes and Genomes pathway analyses, it was determined that 5 pairs of $\operatorname{lncRNAs}$ and their flanking coding genes were simultaneously dysregulated in circulating exosomes. These findings may be valuable for the discovery of potential biomarkers and therapeutic targets for RHD. Wu et al. (94) found that serum exosomal MiR-92b-5p can be used as a potential biomarker for acute HF caused by dilated cardiomyopathy.

Yang et al. (19) used an animal model of cardiac fibrosis and found that with aging, the expression of HSP70 on the surface of exosomes decreased, which may lead to cardiac fibrosis. Zhu et al. (95) found that exosomes derived from human umbilical cord MSCs can prevent senescenceinduced cardiac dysfunction. Further studies have shown that the mechanism may be related to the inhibition of the NF-B/TNF signaling pathway via the release of lncRNA MALAT1. These findings will lead to the development of treatments that delay the progression of aging and agerelated diseases.

\section{Conclusions}

In recent years, more and more studies have been conducted on exosomes and their potential therapeutic applications. 
Current finding support the view that endothelial exosomes may be important initiators of abnormal cell signaling and may be involved in the progression of CVDs. The ability of exosomes to interact with many signaling molecules (including lipids, miRNAs, and proteins) to target specific receptor cells makes them promising diagnostic biomarkers and therapeutic targets for the diagnosis and treatment of CVDs. In fact, exosomes exist in many body fluids and tissues. As a medium of cell-to-cell communication, they participate in anti-inflammatory processes, improve ventricular remodeling, induce cardiac hypertrophy, cardiac fibrosis and promote angiogenesis. They play important roles in cardiovascular physiology and pathology. This review summarized the role of exosomes in the pathogenesis, diagnosis, and treatment of CVDs, and should provide new ideas for the prevention, early diagnosis, and treatment of CVDs and the discovery of biomarkers. The application of exosomes in cardiovascular diseases is still in the preliminary stage of exploration, and there are still many problems that need to be further solved. The future development direction can focus on the following directions: (I) explore the role of exosomes in IncRNA-miRNAprotein and signaling pathways, and further understand the pathogenesis of exosomes in CVD; (II) explore the role of exosomes as biomarkers for disease diagnosis or prognosis assessment in CVD; (III) the therapeutic effects of different stem cell-derived exosomes in CVD have been discovered, but how to target delivery still needs further research.

\section{Acknowledgments}

Funding: This study was supported by the National Natural Science Foundation of China (81804061); the Jinan Science and Technology Project (201805078); the Taishan Scholars Post Construction Funding Project (2018-35).

\section{Footnote}

Reporting Checklist: The authors have completed the Narrative Review reporting checklist. Available at https:// apm.amegroups.com/article/view/10.21037/apm-21-3364/rc

Conflicts of Interest: All authors have completed the ICMJE uniform disclosure form (available at https://apm. amegroups.com/article/view/10.21037/apm-21-3364/coif). All authors report that this study was supported by the National Natural Science Foundation of China (81804061); the Jinan Science and Technology Project (201805078) and the Taishan Scholars Post Construction Funding Project (2018-35). The authors have no other conflicts of interest to declare.

Ethical Statement: The authors are accountable for all aspects of the work in ensuring that questions related to the accuracy or integrity of any part of the work are appropriately investigated and resolved.

Open Access Statement: This is an Open Access article distributed in accordance with the Creative Commons Attribution-NonCommercial-NoDerivs 4.0 International License (CC BY-NC-ND 4.0), which permits the noncommercial replication and distribution of the article with the strict proviso that no changes or edits are made and the original work is properly cited (including links to both the formal publication through the relevant DOI and the license). See: https://creativecommons.org/licenses/by-nc-nd/4.0/.

\section{References}

1. Shpilsky D, Bambs C, Kip K, et al. Association between ideal cardiovascular health and markers of subclinical cardiovascular disease. Clin Cardiol 2018;41:1593-9.

2. The Writing Committee of the Report on Cardiovascular Health and Diseases in China. Interpretation of Report on Cardiovascular Health and Diseases in China 2020. Chinese Journal of Cardiovascular Medicine 2021;26:209-18.

3. Shi C, Ulke-Lemée A, Deng J, et al. Characterization of heat shock protein 27 in extracellular vesicles: a potential anti-inflammatory therapy. FASEB J 2019;33:1617-30.

4. Qiao L, Hu S, Liu S, et al. microRNA-21-5p dysregulation in exosomes derived from heart failure patients impairs regenerative potential. J Clin Invest 2019;129:2237-50.

5. Huang P, Wang L, Li Q, et al. Combinatorial treatment of acute myocardial infarction using stem cells and their derived exosomes resulted in improved heart performance. Stem Cell Res Ther 2019;10:300.

6. Pan BT, Johnstone RM. Fate of the transferrin receptor during maturation of sheep reticulocytes in vitro: selective externalization of the receptor. Cell 1983;33:967-78.

7. Johnstone RM, Adam M, Hammond JR, et al. Vesicle formation during reticulocyte maturation. Association of plasma membrane activities with released vesicles (exosomes). J Biol Chem 1987;262:9412-20.

8. Raposo G, Nijman HW, Stoorvogel W, et al. B lymphocytes secrete antigen-presenting vesicles. J Exp 
Med 1996;183:1161-72.

9. Iwai K, Minamisawa T, Suga K, et al. Isolation of human salivary extracellular vesicles by iodixanol density gradient ultracentrifugation and their characterizations. J Extracell Vesicles 2016;5:30829.

10. Iwai K, Yamamoto S, Yoshida M, et al. Isolation of Extracellular Vesicles in Saliva Using Density Gradient Ultracentrifugation. Methods Mol Biol 2017;1660:343-50.

11. Tschuschke M, Kocherova I, Bryja A, et al. Inclusion Biogenesis, Methods of Isolation and Clinical Application of Human Cellular Exosomes. J Clin Med 2020;9:436.

12. Zhang H, Zhu L, Bai M, et al. Exosomal circRNA derived from gastric tumor promotes white adipose browning by targeting the miR-133/PRDM16 pathway. Int J Cancer 2019; 144:2501-15.

13. Huang L, Zhang X, Wang M, et al. Exosomes from Thymic Stromal Lymphopoietin-Activated Dendritic Cells Promote Th2 Differentiation through the OX40 Ligand. Pathobiology 2019;86:111-7.

14. He S, Li Z, Yu Y, et al. Exosomal miR-499a-5p promotes cell proliferation, migration and EMT via mTOR signaling pathway in lung adenocarcinoma. Exp Cell Res 2019;379:203-13.

15. Hu HY, Yu CH, Zhang HH, et al. Exosomal miR-1229 derived from colorectal cancer cells promotes angiogenesis by targeting HIPK2. Int J Biol Macromol 2019;132:470-7.

16. Ha D, Yang N, Nadithe V. Exosomes as therapeutic drug carriers and delivery vehicles across biological membranes: current perspectives and future challenges. Acta Pharm Sin B 2016;6:287-96.

17. Diao L, Zhang Q. Transfer of lncRNA UCA1 by hUCMSCs-derived exosomes protects against hypoxia/ reoxygenation injury through impairing miR-143targeted degradation of Bcl-2. Aging (Albany NY) 2021;13:5967-85.

18. Li H, Liao Y, Gao L, et al. Coronary Serum Exosomes Derived from Patients with Myocardial Ischemia Regulate Angiogenesis through the miR-939-mediated Nitric Oxide Signaling Pathway. Theranostics 2018;8:2079-93.

19. Yang J, Yu XF, Li YY, et al. Decreased HSP70 expression on serum exosomes contributes to cardiac fibrosis during senescence. Eur Rev Med Pharmacol Sci 2019;23:3993-4001.

20. Chen CW, Fu M, Du ZH, et al. Long Noncoding RNA MRPL23-AS1 Promotes Adenoid Cystic Carcinoma Lung Metastasis. Cancer Res 2020;80:2273-85.

21. Zheng M, Hou L, Ma Y, et al. Exosomal let-7d-3p and miR-30d-5p as diagnostic biomarkers for non-invasive screening of cervical cancer and its precursors. Mol Cancer 2019;18:76.

22. Yu X, Deng L, Wang D, et al. Mechanism of TNF- $\alpha$ autocrine effects in hypoxic cardiomyocytes: initiated by hypoxia inducible factor $1 \alpha$, presented by exosomes. J Mol Cell Cardiol 2012;53:848-57.

23. Loyer X, Zlatanova I, Devue C, et al. Intra-Cardiac Release of Extracellular Vesicles Shapes Inflammation Following Myocardial Infarction. Circ Res 2018;123:100-6.

24. Ribeiro-Rodrigues TM, Laundos TL, Pereira-Carvalho R, et al. Exosomes secreted by cardiomyocytes subjected to ischaemia promote cardiac angiogenesis. Cardiovasc Res 2017;113:1338-50.

25. Nie X, Fan J, Li H, et al. miR-217 Promotes Cardiac Hypertrophy and Dysfunction by Targeting PTEN. Mol Ther Nucleic Acids 2018;12:254-66.

26. Bang C, Batkai S, Dangwal S, et al. Cardiac fibroblastderived microRNA passenger strand-enriched exosomes mediate cardiomyocyte hypertrophy. J Clin Invest 2014;124:2136-46.

27. Lyu L, Wang H, Li B, et al. A critical role of cardiac fibroblast-derived exosomes in activating renin angiotensin system in cardiomyocytes. J Mol Cell Cardiol 2015;89:268-79.

28. van Balkom BW, de Jong OG, Smits M, et al. Endothelial cells require miR-214 to secrete exosomes that suppress senescence and induce angiogenesis in human and mouse endothelial cells. Blood 2013;121:3997-4006, S1-15.

29. Halkein J, Tabruyn SP, Ricke-Hoch M, et al. MicroRNA$146 \mathrm{a}$ is a therapeutic target and biomarker for peripartum cardiomyopathy. J Clin Invest 2013;123:2143-54.

30. Hergenreider E, Heydt S, Tréguer K, et al. Atheroprotective communication between endothelial cells and smooth muscle cells through miRNAs. Nat Cell Biol 2012;14:249-56.

31. Liu Y, Li Q, Hosen MR, et al. Atherosclerotic Conditions Promote the Packaging of Functional MicroRNA-92a-3p Into Endothelial Microvesicles. Circ Res 2019;124:575-87.

32. Davidson SM, Riquelme JA, Zheng Y, et al. Endothelial cells release cardioprotective exosomes that may contribute to ischaemic preconditioning. Sci Rep 2018;8:15885.

33. Hu J, Wang S, Xiong Z, et al. Exosomal Mst1 transfer from cardiac microvascular endothelial cells to cardiomyocytes deteriorates diabetic cardiomyopathy. Biochim Biophys Acta Mol Basis Dis 2018;1864:3639-49.

34. Jansen F, Li Q, Pfeifer A, et al. Endothelial- and Immune Cell-Derived Extracellular Vesicles in the Regulation of 
Cardiovascular Health and Disease. JACC Basic Transl Sci 2017;2:790-807.

35. Akbar N, Digby JE, Cahill TJ, et al. Endotheliumderived extracellular vesicles promote splenic monocyte mobilization in myocardial infarction. JCI Insight 2017;2:e93344.

36. Blaser MC, Aikawa E. Roles and Regulation of Extracellular Vesicles in Cardiovascular Mineral Metabolism. Front Cardiovasc Med 2018;5:187.

37. Kapustin AN, Schoppet M, Schurgers LJ, et al. Prothrombin Loading of Vascular Smooth Muscle Cell-Derived Exosomes Regulates Coagulation and Calcification. Arterioscler Thromb Vasc Biol 2017;37:e22-32.

38. Liu H, Gao W, Yuan J, et al. Exosomes derived from dendritic cells improve cardiac function via activation of CD4(+) T lymphocytes after myocardial infarction. J Mol Cell Cardiol 2016;91:123-33.

39. Wang C, Zhang C, Liu L, et al. Macrophage-Derived mir-155-Containing Exosomes Suppress Fibroblast Proliferation and Promote Fibroblast Inflammation during Cardiac Injury. Mol Ther 2017;25:192-204.

40. Pironti G, Strachan RT, Abraham D, et al. Circulating Exosomes Induced by Cardiac Pressure Overload Contain Functional Angiotensin II Type 1 Receptors. Circulation 2015;131:2120-30.

41. Hou Z, Qin X, Hu Y, et al. Longterm ExerciseDerived Exosomal miR-342-5p: A Novel Exerkine for Cardioprotection. Circ Res 2019;124:1386-400.

42. Cheng M, Yang J, Zhao X, et al. Circulating myocardial microRNAs from infarcted hearts are carried in exosomes and mobilise bone marrow progenitor cells. Nat Commun 2019;10:959.

43. Yao Y, Sun W, Sun Q, et al. Platelet-Derived Exosomal MicroRNA-25-3p Inhibits Coronary Vascular Endothelial Cell Inflammation Through Adam 10 via the NF- $\kappa$ B Signaling Pathway in ApoE-/- Mice. Front Immunol 2019;10:2205.

44. Zhong X, Gao W, Wu R, et al. Dendritic cell exosome-shuttled miRNA146a regulates exosome-induced endothelial cell inflammation by inhibiting IRAK-1: A feedback control mechanism. Mol Med Rep 2019;20:5315-23.

45. Bouchareychas L, Duong P, Covarrubias S, et al. Macrophage Exosomes Resolve Atherosclerosis by Regulating Hematopoiesis and Inflammation via MicroRNA Cargo. Cell Rep 2020;32:107881.

46. Zhang YG, Song Y, Guo XL, et al. Exosomes derived from oxLDL-stimulated macrophages induce neutrophil extracellular traps to drive atherosclerosis. Cell Cycle 2019;18:2674-84.

47. Chen L, Hu L, Li Q, et al. Exosome-encapsulated miR505 from ox-LDL-treated vascular endothelial cells aggravates atherosclerosis by inducing NET formation. Acta Biochim Biophys Sin (Shanghai) 2019;51:1233-41.

48. Li H, Zhu X, Hu L, et al. Loss of exosomal MALAT1 from ox-LDL-treated vascular endothelial cells induces maturation of dendritic cells in atherosclerosis development. Cell Cycle 2019;18:2255-67.

49. Li J, Xue H, Li T, et al. Exosomes derived from mesenchymal stem cells attenuate the progression of atherosclerosis in ApoE-/- mice via miR-let7 mediated infiltration and polarization of M2 macrophage. Biochem Biophys Res Commun 2019;510:565-72.

50. Lin B, Xie W, Zeng C, et al. Transfer of exosomal microRNA-203-3p from dendritic cells to bone marrow-derived macrophages reduces development of atherosclerosis by downregulating Ctss in mice. Aging (Albany NY) 2021;13:15638-58.

51. Liu Y, Sun Y, Lin X, et al. Perivascular Adipose-Derived Exosomes Reduce Foam Cell Formation by Regulating Expression of Cholesterol Transporters. Front Cardiovasc Med 2021;8:697510.

52. Moghaddam AS, Afshari JT, Esmaeili SA, et al. Cardioprotective microRNAs: Lessons from stem cellderived exosomal microRNAs to treat cardiovascular disease. Atherosclerosis 2019;285:1-9.

53. Peng Y, Zhao JL, Peng ZY, et al. Exosomal miR-25$3 \mathrm{p}$ from mesenchymal stem cells alleviates myocardial infarction by targeting pro-apoptotic proteins and EZH2. Cell Death Dis 2020;11:317.

54. Zhu D, Wang Y, Thomas M, et al. Exosomes from adipose-derived stem cells alleviate myocardial infarction via microRNA-31/FIH1/HIF-1 $\alpha$ pathway. J Mol Cell Cardiol 2022;162:10-9.

55. Pan J, Alimujiang M, Chen Q, et al. Exosomes derived from miR-146a-modified adipose-derived stem cells attenuate acute myocardial infarction-induced myocardial damage via downregulation of early growth response factor 1. J Cell Biochem 2019;120:4433-43.

56. Shi Y, Yang Y, Guo Q, et al. Exosomes Derived from Human Umbilical Cord Mesenchymal Stem Cells Promote Fibroblast-to-Myofibroblast Differentiation in Inflammatory Environments and Benefit Cardioprotective Effects. Stem Cells Dev 2019;28:799-811.

57. Santoso MR, Ikeda G, Tada Y, et al. Exosomes From 
Induced Pluripotent Stem Cell-Derived Cardiomyocytes Promote Autophagy for Myocardial Repair. J Am Heart Assoc 2020;9:e014345.

58. Youn SW, Li Y, Kim YM, et al. Modification of Cardiac Progenitor Cell-Derived Exosomes by miR-322 Provides Protection against Myocardial Infarction through Nox2Dependent Angiogenesis. Antioxidants (Basel) 2019;8:18.

59. Yu DW, Ge PP, Liu AL, et al. HSP20-mediated cardiomyocyte exosomes improve cardiac function in mice with myocardial infarction by activating Akt signaling pathway. Eur Rev Med Pharmacol Sci 2019;23:4873-81.

60. Chen Q, Huang $M, W u J$, et al. Exosomes isolated from the plasma of remote ischemic conditioning rats improved cardiac function and angiogenesis after myocardial infarction through targeting Hsp70. Aging (Albany NY) 2020;12:3682-93.

61. Zhang Y, Cai Z, Shen Y, et al. Correction to: Hydrogelload exosomes derived from dendritic cells improve cardiac function via Treg cells and the polarization of macrophages following myocardial infarction. J Nanobiotechnology 2021;19:294.

62. Zhang Y, Cai Z, Shen Y, et al. Hydrogel-load exosomes derived from dendritic cells improve cardiac function via Treg cells and the polarization of macrophages following myocardial infarction. J Nanobiotechnology 2021;19:271.

63. Kurtzwald-Josefson E, Zeevi-Levin N, Rubchevsky V, et al. Cardiac Fibroblast-Induced Pluripotent Stem CellDerived Exosomes as a Potential Therapeutic Mean for Heart Failure. Int J Mol Sci 2020;21:7215.

64. Chen F, Li X, Zhao J, et al. Bone marrow mesenchymal stem cell-derived exosomes attenuate cardiac hypertrophy and fibrosis in pressure overload induced remodeling. In Vitro Cell Dev Biol Anim 2020;56:567-76.

65. Chen Q, Liu Y, Ding X, et al. Bone marrow mesenchymal stem cell-secreted exosomes carrying microRNA-125b protect against myocardial ischemia reperfusion injury via targeting SIRT7. Mol Cell Biochem 2020;465:103-14.

66. Chen G, Wang M, Ruan Z, et al. Mesenchymal stem cellderived exosomal miR-143-3p suppresses myocardial ischemia-reperfusion injury by regulating autophagy. Life Sci 2021;280:119742.

67. Zhao J, Li X, Hu J, et al. Mesenchymal stromal cellderived exosomes attenuate myocardial ischaemiareperfusion injury through miR-182-regulated macrophage polarization. Cardiovasc Res 2019;115:1205-16.

68. Chen L, Wang Y, Pan Y, et al. Cardiac progenitorderived exosomes protect ischemic myocardium from acute ischemia/reperfusion injury. Biochem Biophys Res
Commun 2013;431:566-71.

69. Wang Y, Zhang L, Li Y, et al. Exosomes/microvesicles from induced pluripotent stem cells deliver cardioprotective miRNAs and prevent cardiomyocyte apoptosis in the ischemic myocardium. Int $\mathrm{J}$ Cardiol 2015;192:61-9.

70. Zhang J, Zhang X. Ischaemic preconditioning-induced serum exosomes protect against myocardial ischaemia/ reperfusion injury in rats by activating the PI3K/AKT signalling pathway. Cell Biochem Funct 2021;39:287-95.

71. Dai Y, Wang S, Chang S, et al. M2 macrophage-derived exosomes carry microRNA-148a to alleviate myocardial ischemia/reperfusion injury via inhibiting TXNIP and the TLR4/NF-кB/NLRP3 inflammasome signaling pathway. J Mol Cell Cardiol 2020;142:65-79.

72. Tang J, Jin L, Liu Y, et al. Exosomes Derived from Mesenchymal Stem Cells Protect the Myocardium Against Ischemia/Reperfusion Injury Through Inhibiting Pyroptosis. Drug Des Devel Ther 2020;14:3765-75.

73. Luo H, Li X, Li T, et al. microRNA-423-3p exosomes derived from cardiac fibroblasts mediates the cardioprotective effects of ischaemic post-conditioning. Cardiovasc Res 2019;115:1189-204.

74. Zhang Z, Ge L, Zhang S, et al. The protective effects of MSC-EXO against pulmonary hypertension through regulating Wnt5a/BMP signalling pathway. J Cell Mol Med 2020;24:13938-48.

75. Zhang S, Liu X, Ge LL, et al. Mesenchymal stromal cell-derived exosomes improve pulmonary hypertension through inhibition of pulmonary vascular remodeling. Respir Res 2020;21:71.

76. Zhang M, Xin W, Ma C, et al. Exosomal 15-LO2 mediates hypoxia-induced pulmonary artery hypertension in vivo and in vitro. Cell Death Dis 2018;9:1022.

77. Sindi HA, Russomanno G, Satta S, et al. Therapeutic potential of KLF2-induced exosomal microRNAs in pulmonary hypertension. Nat Commun 2020;11:1185.

78. Tong Y, Ye C, Ren XS, et al. Exosome-Mediated Transfer of ACE (Angiotensin-Converting Enzyme) From Adventitial Fibroblasts of Spontaneously Hypertensive Rats Promotes Vascular Smooth Muscle Cell Migration. Hypertension 2018;72:881-8.

79. Otani K, Yokoya M, Kodama T, et al. Plasma exosomes regulate systemic blood pressure in rats. Biochem Biophys Res Commun 2018;503:776-83.

80. Zhu Z, Shen Y, Chen Y, et al. The exosome of platelet endothelial cell adhesion molecule-1 (PECAM1) protein: A potential risking star in high blood pressure patients 
(HBPP). Medicine (Baltimore) 2021;100:e21370.

81. Perez-Hernandez J, Olivares D, Forner MJ, et al. Urinary exosome miR-146a is a potential marker of albuminuria in essential hypertension. J Transl Med 2018;16:228.

82. Perez-Hernandez J, Riffo-Campos AL, Ortega A, et al. Urinary- and Plasma-Derived Exosomes Reveal a Distinct MicroRNA Signature Associated With Albuminuria in Hypertension. Hypertension 2021;77:960-71.

83. Wei Z, Bing Z, Shaohuan Q, et al. Expression of miRNAs in plasma exosomes derived from patients with atrial fibrillation. Clin Cardiol 2020;43:1450-9.

84. Mun D, Kim H, Kang JY, et al. Expression of miRNAs in circulating exosomes derived from patients with persistent atrial fibrillation. FASEB J 2019;33:5979-89.

85. Li S, Gao Y, Liu Y, et al. Myofibroblast-Derived Exosomes Contribute to Development of a Susceptible Substrate for Atrial Fibrillation. Cardiology 2020;145:324-32.

86. Liu L, Zhang H, Mao H, et al. Exosomal miR-320d derived from adipose tissue-derived MSCs inhibits apoptosis in cardiomyocytes with atrial fibrillation (AF). Artif Cells Nanomed Biotechnol 2019;47:3976-84.

87. Ma LY, Chen WW, Gao RL, et al. China cardiovascular diseases report 2018: an updated summary. J Geriatr Cardiol 2020;17:1-8.

88. Govindappa PK, Patil M, Garikipati VNS, et al. Targeting exosome-associated human antigen $\mathrm{R}$ attenuates fibrosis and inflammation in diabetic heart. FASEB J 2020;34:2238-51.

89. Lin Y, Zhang F, Lian XF, et al. Mesenchymal stem cellderived exosomes improve diabetes mellitus-induced

Cite this article as: Cui M, Han Y, Yang J, Li G, Yang C. A narrative review of the research status of exosomes in cardiovascular disease. Ann Palliat Med 2022;11(1):363-377. doi: 10.21037/apm-21-3364 myocardial injury and fibrosis via inhibition of TGF- $\beta 1 /$ Smad2 signaling pathway. Cell Mol Biol (Noisy-le-grand) 2019;65:123-6.

90. Gu X, Li Y, Chen K, et al. Exosomes derived from umbilical cord mesenchymal stem cells alleviate viral myocarditis through activating AMPK/mTOR-mediated autophagy flux pathway. J Cell Mol Med 2020;24:7515-30.

91. Li X, Yang Z, Nie W, et al. Exosomes derived from cardiac progenitor cells attenuate $\mathrm{CVB} 3$-induced apoptosis via abrogating the proliferation of CVB3 and modulating the mTOR signaling pathways. Cell Death Dis 2019;10:691.

92. Chen A, Wen J, Lu C, et al. Inhibition of miR-155-5p attenuates the valvular damage induced by rheumatic heart disease. Int J Mol Med 2020;45:429-40.

93. Luo Y, Huang L, Luo W, et al. Genomic analysis of lncRNA and mRNA profiles in circulating exosomes of patients with rheumatic heart disease. Biol Open 2019;8:bio045633.

94. Wu T, Chen Y, Du Y, et al. Serum Exosomal MiR-92b$5 \mathrm{p}$ as a Potential Biomarker for Acute Heart Failure Caused by Dilated Cardiomyopathy. Cell Physiol Biochem 2018;46:1939-50.

95. Zhu B, Zhang L, Liang C, et al. Stem Cell-Derived Exosomes Prevent Aging-Induced Cardiac Dysfunction through a Novel Exosome/lncRNA MALAT1/NF$\kappa \mathrm{B} / \mathrm{TNF}-\alpha$ Signaling Pathway. Oxid Med Cell Longev 2019;2019:9739258.

(English Language Editor: L. Huleatt) 